de Dios, O. G., Casellas, R., Paolucci, F., Napoli, A., Gifre, L., Annoni, S., Belotti, S., Feiste, U., Rafique, D., Bohn, M., Bigo, S., Dupas, A., Dutisseuil, E., Fresi, F., Guo, B., Hugues Salas, E., Layec, P., López, V., Meloni, G., ... Fernández-Palacios, J. (2015). First demonstration of multi-vendor and multi-domain EON with S-BVT and control interoperability over Pan-European testbed. In 2015 European Conference on Optical Communication (ECOC 2015): Proceedings of a meeting held 27 September - 1 October 2015, Valencia, Spain [7341689] Institute of Electrical and Electronics Engineers (IEEE). https://doi.org/10.1109/ECOC.2015.7341689

Peer reviewed version

Link to published version (if available):

10.1109/ECOC.2015.7341689

Link to publication record in Explore Bristol Research

PDF-document

University of Bristol - Explore Bristol Research

General rights

This document is made available in accordance with publisher policies. Please cite only the published version using the reference above. Full terms of use are available:

http://www.bristol.ac.uk/red/research-policy/pure/user-guides/ebr-terms/ 


\title{
First Demonstration of Multi-vendor and Multi-domain EON with S-BVT and Control Interoperability over Pan-European Testbed
}

\author{
O. Gonzalez de Dios ${ }^{(1)}$, R. Casellas ${ }^{(2)}$, F. Paolucci ${ }^{(3),}$ A. Napoli ${ }^{(4)}$, LI. Gifre ${ }^{(5)}$, S. Annoni $i^{(6)}$, S. Belotti ${ }^{(6)}$,

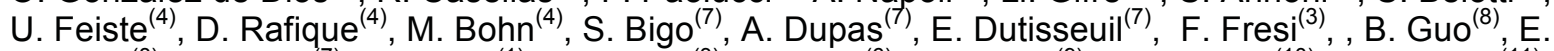

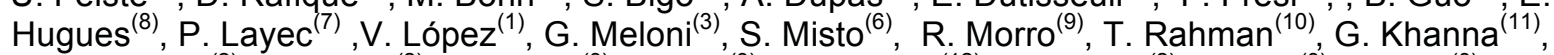 \\ R. Martínez ${ }^{(2)}$, R. Vilalta ${ }^{(2)}$, F. Cugini ${ }^{(3)}$, L. Poti ${ }^{(3)}$, A. D'Errico ${ }^{(12)}$, R. Muñoz $^{(2)}, Y$. Shu $^{\left({ }^{\prime}\right)}$, S. Yan ${ }^{(8)}$, Y.' \\ Yan $^{(8)}$, G. Zervas ${ }^{(8)}$, R. Nejabati ${ }^{(8)}$, D. Simeonidou ${ }^{(8)}$, L. Velasco ${ }^{(5)}$ and J. Fernández-Palacios ${ }^{(1)}$ \\ (1) Telefónica I+D, Spain, email:oscar.gonzalezdedios@telefonica.com ${ }^{(2)}$ CTTC, Spain, ${ }^{(3)}$ CNIT- \\ Scuola Superiore Sant' Anna, Italy, ${ }^{(4)}$ Coriant R\&D, Germany, ${ }^{(5)}$ UPC GCO, Spain ${ }^{(6)}$ Alcatel-Lucent, \\ Italy, ${ }^{(7)}$ Alcatel-Lucent, France, ${ }^{(8)}$ University of Bristol, UK, ${ }^{(9)}$ Telecom Italia, Italy, ${ }^{(10)}$ TU/e Eindhoven, \\ Netherlands, ${ }^{(11)}$ Technische Universität München, Germany, ${ }^{(12)}$ Ericsson Research, Italy
}

Abstract The operation of multi-domain and multi-vendor EONs can be achieved by interoperable Sliceable Bandwidth Variable Transponders, a GMPLS / BGP-LS-based control plane and a planning tool. This paper reports the first full demonstration and validation this end-to-end architecture.

\section{Introduction}

Elastic Optical Networks (EONs) are envisioned as the base of next generation transport networks. However, its commercial deployment requires the data and control solutions to interoperate among different implementations.

In this paper, we present, for the first time, an integrated multi-domain, multi-vendor, flexi-grid, control and data plane network. Protocol extensions are evaluated in a distributed multipartner control plane test-bed, fully enabling the interoperability of several implementations of Sliceable Bandwidth Variable Transponders (SBVTs), which are experimentally validated.

Network architecture and control procedures The EON network envisioned by the EU IDEALIST project is based on software configurable Flexi-grid optical Switching, SBVTs and elastic OTN grooming. The control architecture of such network and the interfaces (Fig.1 left) extend the one previously presented ${ }^{1}$ in order to include the control of S-BVTs. A hierarchical Path Computation Element (H-PCE) is deployed for real-time path computation and provisioning, with the parent PCE (pPCE), under guidance from the ABNO controller, coordinating the procedures between child PCEs (CPCE) deployed at each domain with BGP Link-State speakers (Fig. 1). The latter is used to disseminate, between the CPCEs and PPCE, abstracted view of the intra-domain network along with the Traffic Engineering (TE) attributes of the inter-domain links. The interface between PPCE and domain cPCEs (based on PCEP protocol) is used to instantiate connections or Label Switched Paths (LSPs), since each domain deploys a GMPLS control plane (OSPFTE for routing and topology dissemination, RSVP-TE for signalling) and configures the underlying hardware.

The specialized off-line planning tool ${ }^{4}$ uses BGP-LS updates to obtain the intra-domain topology, including the capabilities of multi-flow transponders of the different vendors, and performs CPU-intensive computations. The result of the planning process, upon approval of the network operator, is sent to the ABNO controller, which sends PCEP Initiate Requests to the PPCE, which coordinates the instantiation in each domain. The network offers the provisioning and recovery of transport flexi-grid media channels or, where appropriate, end-toend electrical connections with different bit rates with dynamic ODUFlex grooming over BVTs.

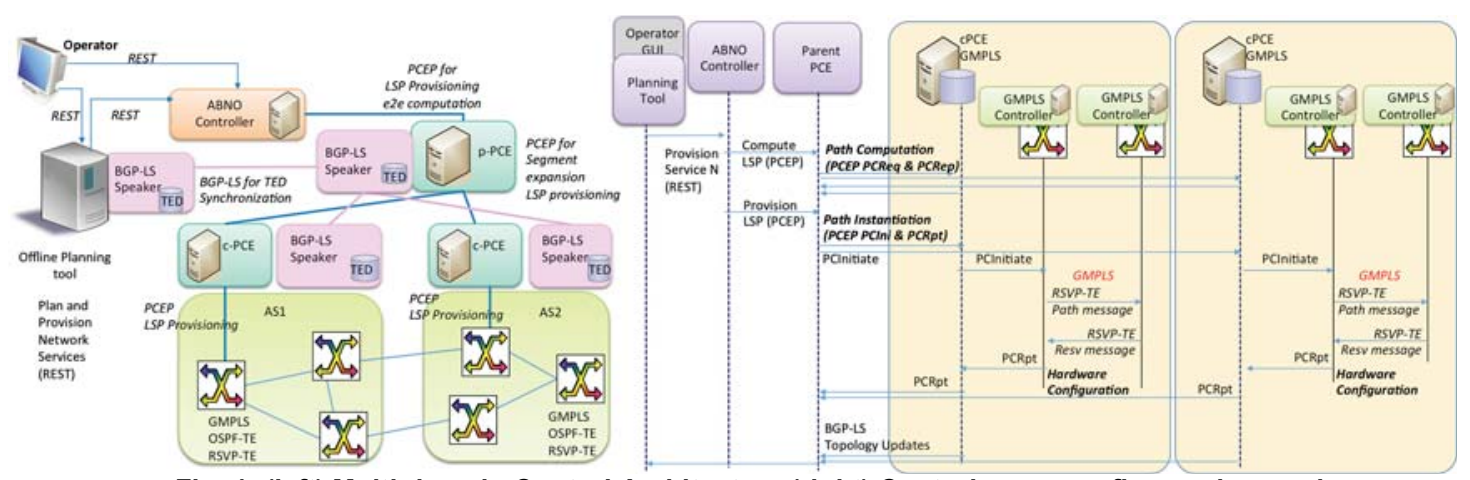

Fig. 1: (left) Multi-domain Control Architecture (right) Control message flow and procedures 


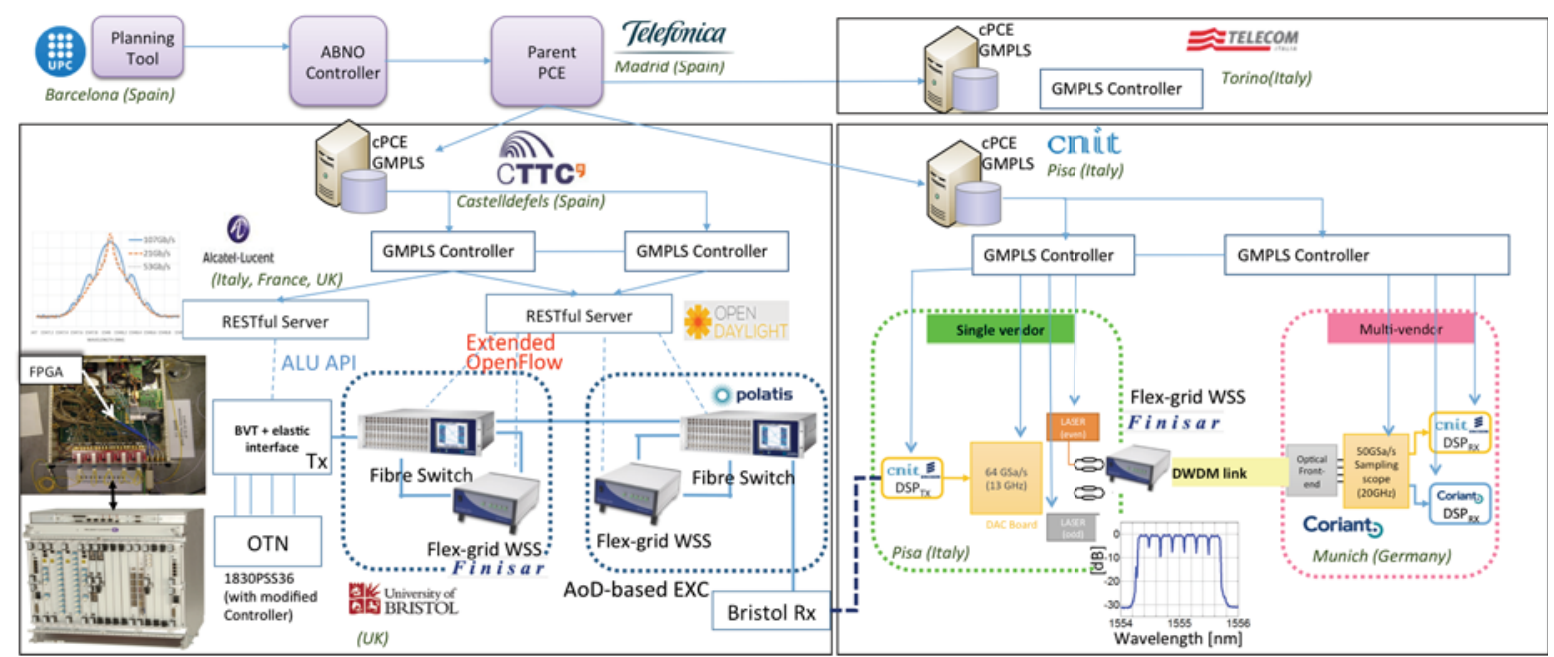

Fig. 2: IDEALIST Multi-partner Pan-European Testbed integrating control and data

\section{Multi-Partner Testbed Description}

The testbed, shown in Fig. 2, is built by the interconnection of different components, both hardware and software, physically distributed within labs. The testbed encompasses three Flexi-grid domains with different capabilities, one hierarchical PCE, an ABNO Controller and the PLATON planning tool. The domains are interconnected resulting in the inter-domain topology shown in Fig. 3.

The first flexi-grid domain (102) is composed of two nodes (Fig. 2 bottom-left). Each node includes: (I) a flexible and configurable digital cross connect (OTN fabric) for client mapping and centralized control of S-BVT; (II) real-time S-BVT modules carrying multiple OTU2 tributaries thanks to programmable FPGAs and a (multi-flow) optical front end that can adapt its symbol rate such as $107 \mathrm{~Gb} / \mathrm{s}, 53.5 \mathrm{~Gb} / \mathrm{s}$ per carrier $^{2}$ and its number of carriers according to the reach, physical impairments or capacity demand; (III) an optical matrix that provides flexibility through architecture-on-demand (AoD) in terms of synthesis of fibre switching crossconnections, optical bandwidth switching with bandwidth variable WSS ${ }^{3}$.

The GMPLS control plane is thus able to configure the underlying hardware (via the connection control interface) by using dedicated

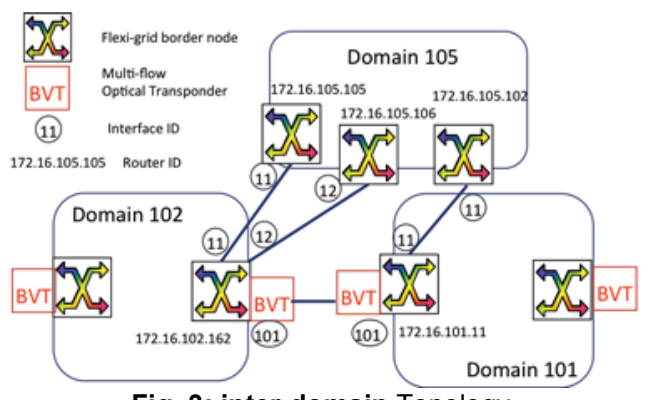

Fig. 3: inter-domain Topology
REST interfaces, as mandated by the signalling process. This covers the cross-connection configuration, the WSS filters and the S-BVT. A deployed middleware translates the high-level REST interfaces to the actual low-level hardware interface. In particular, it can configure the number of physical OTU2 tributaries/lanes interfaced to the elastic BVT, and the required symbol-rate. So if there are five OTU2 lanes to be carried, the BVT is configured at $13 \mathrm{GBd}$ PDM-QPSK. The middleware contain RESTful Servers e.g. running as a northbound interface on top of a SDN OpenDayLight (ODL) controller with their southbound interface based on extended OpenFlow Protocol (OFP). The transport equipment (e.g., Fibre Switch and BVWSS) has an OFP agent capable to translate the received OFP commands to specific equipment APIs (e.g., TL1).

The second domain (101) (Fig. 2 bottom right) comprises integrated data and control plane. The data plane setup consists of 4 nodes flexgrid network, a CNIT /Ericsson DSP unit at the TX and two different ones (CNIT/Ericsson and Coriant) at the RX as part of an optical coherent test-bed. The TX is able to provide a super-channel with different number of carriers and capacity (i.e. 1 carrier for 100G, 3 carriers for $400 \mathrm{G}$ and 7 carriers for $1 \mathrm{~T}$ ). At $1 \mathrm{~Tb} / \mathrm{s}$, we adopted PM-16QAM Nyquist-shaped signals shaped by a roll-off $=0.05$ and symbol rate $=$ 23GBd. A 64 GSa/s Digital-to-Analog Converter (DAC) was used with digital pre-emphasis to compensate for its bandwidth limitations. Subcarrier spacing was set to $25 \mathrm{GHz}$. The testbed was equipped with an optical re-circulating loop consisting of $2 \times 80 \mathrm{~km}$ SSMF spans, EDFAs to compensate for span losses and a waveshaper acting as a loop filter to perform gain equalization and suppress accumulated out of 

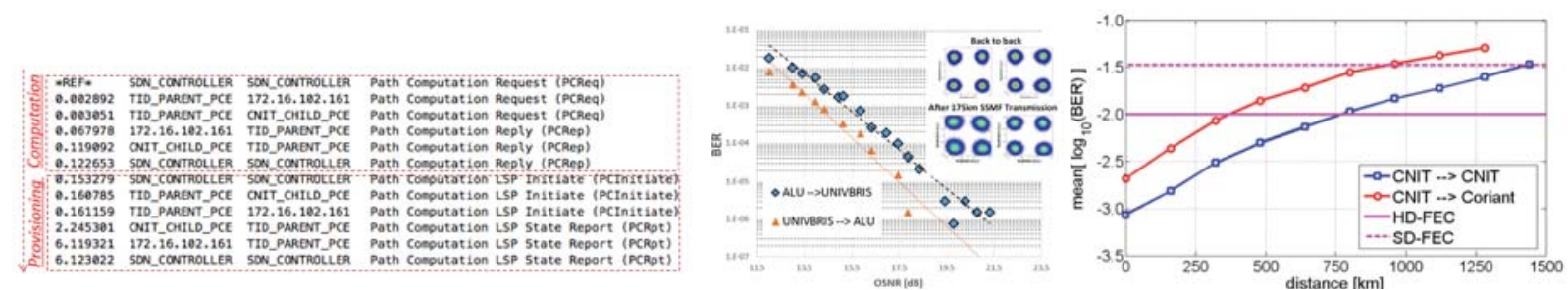

Fig. 4: (left) Message Capture at pPCE (center) Back-to-back BERvsOSNR performance (ALU/UNIVBRIS), constellations for B2B/175km transmission through 2 nodes using the elastic SBVT (right) Single- and cross-vendor transmission reach

band ASE noise. After the loop circulation, the desired sub-carrier selected by filtered and demodulated by employing coherent phase- and polarization-diversity detection and setting the local oscillator (LO) at the nominal wavelength of the selected sub-carrier. The received optical signal is sampled and digitized at $50 \mathrm{GSa} / \mathrm{s}$ with a $20 \mathrm{GHz}$ real-time oscilloscope for off-line postprocessing and BER measurements. Control is performed via GMPLS controllers running RSVP-TE and PCEP and dynamically configuring BV-WSS, transponders (i.e., symbol rate, carriers, frequencies, etc.) and DSP parameters (i.e., modulation, FEC, etc.). Interoperability between CNIT/Ericsson and Coriant DSPs is achieved by the exchange of novel Transponder Class attributes in BGP-LS and PCEP/RSVP-TE protocols, including agreed ranges of physical parameters. Path computation allows transponder-transponder paths only if they belong to the same class.

The domain (105) is composed of six nodes running a GMPLS control plane performing data plane emulation at the media layer; no data plane devices are deployed. The PPCE and ABNO Controller are java-based applications performing multi-domain computation and orchestration, enabling multiple workflows.

\section{Experimental Evaluation}

To demonstrate the architecture, an end-to-end connection of $107 \mathrm{~Gb} / \mathrm{s}$, with one segment in domain 102, and a second segment with a cross-vendor connection between S-BVTs in domain 101 , is setup. A Wireshark capture of the PCEP messages flow of the computation and instantiation is shown in Fig. 4 left, with a total set-up time of 6.12 seconds. Fig. 4 center shows the resulting BER vs. OSNR curves of the elastic SBVT interfaces and DSP receivers from Alcatel-Lucent and University of Bristol. For ALU $\rightarrow$ UNIVBRIS, ALU's transmitter is used as well as the DSP of UNIVBRIS at the receiver. Conversely, for UNIVBRIS $\rightarrow$ ALU, the SBVT transmitter of UNIVBRIS is used and the DSP design of $A L U$. A minimum penalty of $\angle 2 d B$ is observed in the curves, showing the effectiveness of interoperability in between different elastic SBVTs. The figure shows constellations for the back-to-back and aftertransmission cases including $175 \mathrm{~km}$ of SSMF and two AoD nodes. The results show the interoperability between the ALU SBVT transmitter and UNIVBRIS SBVT receiver. Fig. 4 right reports the transmission performance of the single-vendor (CNIT) \& cross-vendor (CNIT/Coriant) solution, which is deployed in the 101 domain at the maximum capacity. The line rate of $1.28 \mathrm{~Tb} / \mathrm{s}$ allows a $23 \% \mathrm{FEC}-\mathrm{OH}$ plus $5 \%$ framing $\mathrm{OH}$. Under this assumption, the preFEC BER threshold (BER $R_{\text {th }}$ ) of $3.4 \times 10^{-2}$ can be considered for the single-vendor solution, as more powerful data-aided vendor-specific SDFEC can be adopted ${ }^{5,6}$. This provides a transmission distance $\geq 1250 \mathrm{~km}$ for the singlevendor solution. If the cross-vendor one is adopted, with standard hard-decision (HD) FEC and blind DSP processing, the $\mathrm{BER}_{\mathrm{th}} 1 \times 10^{-2}$ can be considered, reducing the max distance to $\sim 300 \mathrm{~km}$.

\section{Conclusions}

We demonstrated for the first time a fully end-toend interoperable EON network at control and data plane levels. The control architecture configures SBVTs so the multi-vendor transmission reach is failure-free up to $300 \mathrm{~km}$.

\section{Acknowledgements}

This work was fully supported by FP7 EU project IDEALIST under grant agreement $n^{\circ} 317999$.

\section{References}

[1] O. González de Dios et al. "First Multi-partner Demonstration of BGP-LS enabled Inter-domain EON control with H-PCE", OFC 2015

[2] A. Dupas et al. "Real-time Demonstration of Software defined Elastic Interface for Flexgrid Networks, OFC2015, Los Angeles USA, March 22-26th 2015.

[3] E. Hugues-Sales et al., "Next Generation Optical Nodes: The Vision of the European Research Project IDEALIST IEEE Comm. Magazine. February 2015. Pp. 172-181.

[4] L. Velasco et al, "iONE: A Workflow-Oriented ABNO Implementation" Proc. IEEE/OSA P. in Switching, 2015.

[5] M. Gunkel et al. "Elastic Black Link for Future Vendor Independent Optical Networks." OFC, 2015, pp. Th1I-3.

[6] M. Kuschnerov et al. "Data-aided versus blind singlecarrier coherent receivers."IEEE Photonics Journal 2010 